International Mathematical Forum, 1, 2006, no. 33, 1635-1644

\title{
O.R. Theory and it's Application Multi-Component Commercial Bank Branch Progress and Regress: An Application of Data Envelopment Analysis
}

\author{
A. Divandari \\ Department of Management, Tehran University, Tehran, Iran \\ G. R. Jahanshahloo and F. Hosseinzadeh Lotfi ${ }^{1}$ \\ Department of Mathematics, Science and Research Branch \\ Islamic Azad University, Tehran, Iran ${ }^{2}$

\section{H. Nikoomaram} \\ Department of Management, Science and Research Branch \\ Islamic Azad University, Tehran, Iran \\ F. Rezai Balf \\ Department of Mathematics, Islamic Azad University, Qaemshahr, Iran
}

\begin{abstract}
The purpose of the present study is to develop a method used to evaluate the progress and regress of cammercial bank different branches during different periods. It has to be mentioned that data envelopment analysis is used to develop such a method. The results seem to be quite satisfactory from managerial point of view.
\end{abstract}

Mathematics Subject Classification: Operations Research, 90. Keywords: Data Envelopment Analysis, Progress, Regress

\footnotetext{
${ }^{1}$ Corresponding author, Email addresses: hosseinzadeh_lotf@@yahoo.com

${ }^{2}$ Tel.:+98-21-44804172, Fax: +98-21-44804172, P.O. Box 14155/775 and 14155/4933, Post code: 1477893855
} 


\section{Introduction}

In evaluating the relative efficiency of a set of decision making units, you may prefer to compare the results in two separate periods to find out their progress and regress. Data envelopment analysis is used for this purpose. Considering a special function, it seems that the results are very realistic and satisfactory from managerial point of view. The regress and progress of different components for different branches enables the manager to take relevant decisions for these components. This paper contains following sections: in section 2 basic concepts are explained, the proposed model for calculating progress and regress is put forward in section 3, and the application of this method in Bank Mellat branches, are discussed in the last section is conclusion.

\section{Background}

Suppose that, $\left\{D M U_{j}: j=1, \ldots, n\right\}$ is a set of decision-making units, and $D M U_{j}$ consumes $m$ input $X=\left(x_{1 j}, \ldots, x_{m j}\right)$ to produce $s$ output $Y=$ $\left(y_{1 j}, \ldots, y_{s j}\right)$ as non-negative amounts, and $X \geq 0, Y \geq 0, X \neq 0, Y \neq 0$.

The production possibility set (PPS) is created in association with postulates, non-empty, constant returns to scale, convexity and plausibility as follow:

$T_{c}=\left\{(X, Y): X \geq \sum_{j}^{n} \lambda_{j} X_{j}, Y \leq \sum_{j}^{n} \lambda_{j} Y_{j}\right\}$

The frontier created with each production possibility set is called the respondent efficiency of PPS. To assess efficient units, their distance from boarder is measured. If it is located on the frontier it is called an efficient unit, otherwise it would be inefficient. To find out if $D M U_{p}$ is located on the efficiency frontier or not, the following linear programming problem is solved.

$$
\begin{aligned}
\operatorname{Max} & z=U^{T} Y_{p} \\
\text { s.t. } & U^{T} Y_{j}-V^{T} X_{j} \leq 0, \quad j=1, \ldots, n \\
& V^{T} X_{p}=1 \\
& U, V \geq \overrightarrow{1} \epsilon
\end{aligned}
$$

If in optimal solution (1), $z_{p}^{*}=1$, then $D M U_{p}$ is efficient otherwise it would be inefficient. ( In this paper radial efficiency is considered). Now, consider that each decision making unit consists of two components. Suppose that, $X^{1}$ is the specific input vector for the first component, $X^{2}$ is specific input vector for the second component, $\bar{X}$ is the shared input vector for both components, $Y^{1}$ is the specific output vector for the first component, $Y^{2}$ is the specific output vector for the second component, and $\bar{Y}$ is the shared output vector for both components.

Let $\alpha \bar{X},(1-\alpha) \bar{X}$ are the vectors of shared input and $\beta \bar{Y},(1-\beta) \bar{Y}$ are share output of component 1 and 2 respectively. The aggregated efficiency and component efficiency is obtained by solving the following problem. 


$$
\begin{gathered}
\text { Max } \quad e_{p}^{a}=\frac{U^{1} Y_{p}^{1}+\bar{U}^{1}\left(\beta \bar{Y}_{p}\right)+U^{2} Y_{p}^{2}+\bar{U}^{2}(1-\beta) \bar{Y}_{p}}{V^{1} X_{p}^{1}+V^{1}\left(\alpha \bar{X}_{p}\right)+V^{2} X_{p}^{2}+V^{2}(1-\alpha) \bar{X}_{p}} \\
e_{j}^{1}=\frac{U^{1} Y_{j}^{1}+\bar{U}^{1}\left(\beta \bar{Y}_{j}\right)}{V^{1} X_{j}^{1}+\bar{V}^{1}\left(\alpha \bar{X}_{j}\right)} \leq 1, \quad j=1, \ldots, n \\
e_{j}^{2}=\frac{U^{2} Y_{j}^{2}+\bar{U}^{2}(1-\beta) \bar{Y}_{j}}{V^{2} X_{j}^{2}+\bar{V}^{2}(1-\alpha) \bar{X}_{j}} \leq 1, \quad j=1, \ldots, n \\
U^{1} \geq \overrightarrow{1} \epsilon, U^{2} \geq \overrightarrow{1} \epsilon, \bar{U}^{1} \geq \overrightarrow{1} \epsilon, \bar{U}^{2} \geq \overrightarrow{1} \epsilon \\
V^{1} \geq \overrightarrow{1} \epsilon, V^{2} \geq \overrightarrow{1} \epsilon, \bar{V}^{1} \geq \overrightarrow{1} \epsilon, \bar{V}^{2} \geq \overrightarrow{1} \epsilon
\end{gathered}
$$

Consider the substitutions

$\bar{U}^{1} \beta=U^{3}, \bar{U}^{2}(1-\beta)=U^{4}, \bar{V}^{1} \alpha=V^{3}, \bar{V}^{(1-\alpha)}=V^{4}$,

We can transform problem (2) as linear form follows:

$$
\begin{aligned}
\text { Max } & z=U^{1} Y_{p}^{1}+U^{2} Y_{p}^{2}+U^{3} \bar{Y}_{p}+U^{4} \bar{Y}_{p} \\
\text { s.t. } & V^{1} X_{p}^{1}+V^{2} X_{p}^{2}+V^{3} \bar{X}_{p}+V^{4} \bar{X}_{p}=1 \\
& U^{1} Y_{j}^{1}+U^{3} \bar{Y}_{j}-V^{1} X_{j}^{1}-V^{3} \bar{X}_{j} \leq 0, \quad j=1, \ldots, n \\
& U^{2} Y_{j}^{2}+U^{4} \bar{Y}_{j}-V^{2} X_{j}^{2}-V^{4} \bar{X}_{j} \leq 0, \quad j=1, \ldots, n \\
& U^{1} \geq \overrightarrow{1} \epsilon, U^{2} \geq \overrightarrow{1} \epsilon, U^{3} \geq \overrightarrow{1} \beta \epsilon, U^{4} \geq \overrightarrow{1}(1-\beta) \epsilon \\
& V^{1} \geq \overrightarrow{1} \epsilon, V^{2} \geq \overrightarrow{1} \epsilon, V^{3} \geq \overrightarrow{1} \alpha \epsilon, V^{4} \geq \overrightarrow{1}(1-\alpha) \epsilon
\end{aligned}
$$

The optimal solution model (3) the efficiency of the first, second, and aggregate components by using the following formulas:

Efficiency of the first component $=e_{p}^{1}=\frac{U^{1} Y_{p}^{1}+U^{3} \bar{Y}_{p}}{V^{1} X_{p}^{1}+V^{3} \bar{X}_{p}}$

Efficiency of the second component $=e_{p}^{2}=\frac{U^{2} Y_{p}^{2}+U^{4} \bar{Y}_{p}}{V^{2} X_{p}^{2}+V^{4} \bar{X}_{p}}$

Efficiency of the aggregate component $=e_{p}^{a}=\frac{U^{1} Y_{p}^{1}+U^{3} \bar{Y}_{p}+U^{2} Y_{p}^{2}+U^{4} \bar{Y}_{p}}{V^{1} X_{p}^{1}+V^{3} X_{p}+V^{2} X_{p}^{2}+V^{4} X_{p}}$

\section{Progress and Regress of Components}

Suppose that we are going to assess the situation of a DMU in two different periods of time or we want to compare its improvement in these two periods. Since the relative efficiency of each period is calculated according to that specific period, assessing the relative efficiency of each unit in two independent periods of time doesn't give us the accurate information about their progress and regress. Suppose that a decision making unit, such as, was relatively efficient in first period and also proved efficient in second period, compared with all units. Whereas the value of its efficiency in both periods is 1 , no progress and no regress occur, but we don't know if the efficiency frontier, relative to the previous period, is in better situation or worse. 
Here, a method is proposed to solve this problem. To measure the amount of progress and regress in two periods of time, just compare the efficiency of the second period with the efficiency frontier of the first period, or we can say, compare its relative efficiency with the previous efficiency frontier, in this way the efficiency of the first and second periods are compared with each other and we obtain a criteria to evaluate the progress and regress of the units. Consider the following figure:

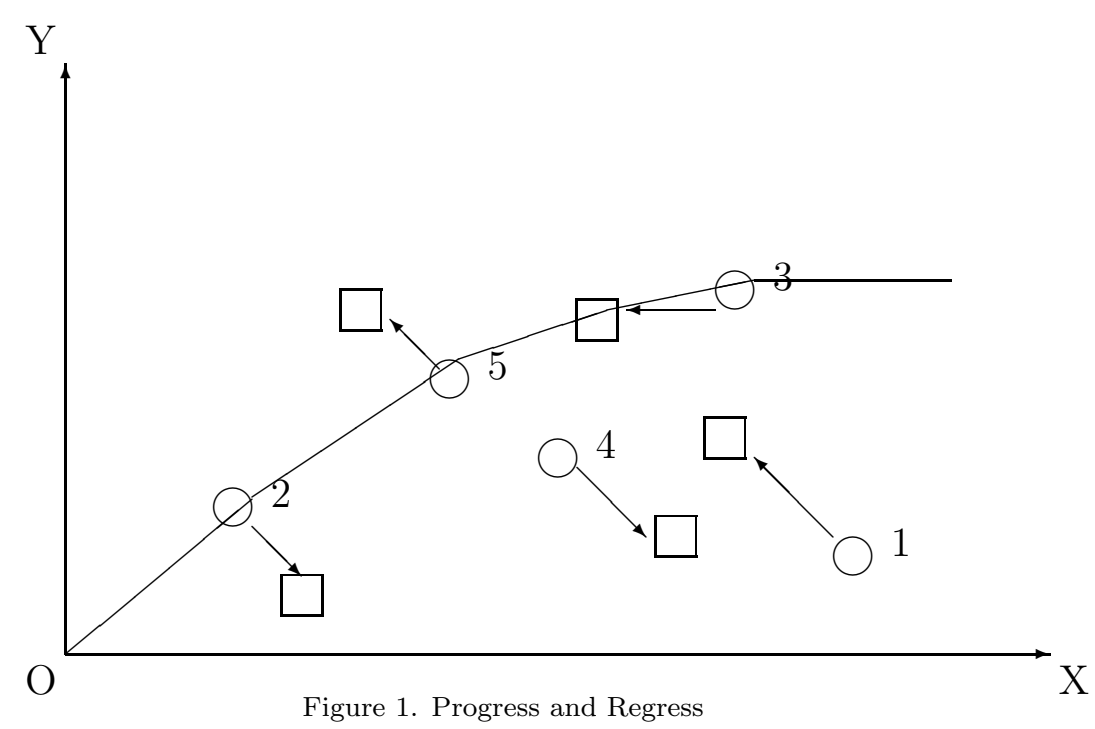

In Figure 1, you see five decisions making unites produce an output using single input. Circles show the situation of these units in the first period, and squares show their situation in the second period. The frontier is the efficiency frontier in the first period. The efficiency of each unit can be determined by measuring its distance from the frontier, nearer units is more efficient. For example, unit 5 in the second period is nearer to the frontier than the first period, so this unit has made progress, and in this way, unit 4 shows regress. Generally, in Figure 1, unites 1 and 5 made progress and units 2 and 4 made regress, and unit 3 remains in the same situations without progress and regress. To assess the progress and regress of a unit, apply the above mentioned procedure to it. Now, consider that we are going to measure the progress and regress of these unites in two periods of time and compare the changes of a period with the changes of another period, for example we compare this summer with last summer and suppose that the data is gathered monthly.

The first step is calculating the efficiency of each decision making unit in the first period. The following model calculates the efficiency of each component of $D M U_{p}$ in the period $\mathrm{t}=1, \ldots, \mathrm{T}$. 


$$
\begin{aligned}
\operatorname{Max} & Z=\sum_{t}^{T} U^{1 t} Y_{p}^{1 t}+U^{2 t} Y_{p}^{2 t}+U^{3 t} \bar{Y}_{p}^{t}+U^{4} \bar{Y}_{p}^{t} \\
\text { s.t. } & V^{1 t} X_{p}^{1 t}+V^{2 t} X_{p}^{2 t}+V^{3 t} \bar{X}_{p}^{t}+V^{4} \bar{X}_{p}^{t}=1 \\
& U^{1 t} Y_{j}^{1 t}+U^{3 t} \bar{Y}_{j}^{t}-V^{1 t} X_{j}^{1 t}-V^{3 t} \bar{X}_{j}^{t} \leq 0, \quad j=1, \ldots, n \\
& U^{2 t} Y_{j}^{2 t}+U^{4 t} \vec{Y}_{j}^{t}-V^{2 t} X_{j}^{2 t}-V^{4 t} \bar{X}_{j}^{t} \leq 0, \quad j=1, \ldots, n \\
& U^{1 t} \geq \overrightarrow{1} \epsilon, U^{2 t} \geq \overrightarrow{1} \epsilon, U^{3 t} \geq \overrightarrow{1} \beta \epsilon, U^{4 t} \geq \overrightarrow{1}(1-\beta) \epsilon, \quad t=1, \ldots, T \\
& V^{1 t} \geq \overrightarrow{1} \epsilon, V^{2 t} \geq \overrightarrow{1} \epsilon, V^{3 t} \geq \overrightarrow{1} \alpha \epsilon, V^{4 t} \geq \overrightarrow{1}(1-\alpha) \epsilon, \quad t=1, \ldots, T .
\end{aligned}
$$

By solving problem (5), accumulated efficiency of $D M U_{p}$ in the total period and the efficiency of the first and second components in each t period would be calculated as follows:

The efficiency of the first component in stage $t=e_{p}^{1 t}=\frac{U^{1 t} Y_{p}^{1 t}+U^{3 t} \bar{Y}_{p}^{t}}{V^{1 t} X_{p}^{1 t}+V^{3 t} \bar{X}_{p}^{t}}$

The efficiency of the second component in stage $t=e_{p}^{2 t}=\frac{U^{2 t} Y_{p}^{2 t}+U^{4 t} \bar{Y}_{p}^{t}}{V^{2 t} X_{p}^{2 t}+V^{4 t} X_{p}^{t}}$

The efficiency of the aggregate component in stage

$t=e_{p}^{a t}=\frac{U^{1 t} Y_{p}^{1 t}+U^{3 t} \bar{Y}_{p}^{t}+U^{2 t} Y_{p}^{2 t}+U^{4 t} \bar{Y}_{p}^{t}}{V^{1 t} X_{p}^{1 t}+V^{3 t} \bar{X}_{p}^{t}+V^{2 t} X_{p}^{2 t}+V^{4 t} \bar{X}_{p}^{t}}$

Now, according to the proposed model, in the new period, each decision making unit would be compared with all units of the first period which were efficient in at least one component and at least in one single $t$. Suppose $e_{j}^{\prime}$ is the new efficiency ( in the second duration) and $e_{j}$ is the old one (in the first duration), thus:

Where $e_{j}^{\prime}>e_{j}, D M U_{j}$ shows progress.

Where $e_{j}^{\prime}=e_{j}$, there isn't any progress and regress.

Where $e_{j}^{\prime}<e_{j}, D M U_{j}$ shows regress.

Now, we are going to propose a criterion for measuring progress and regress.

Consider that the proportion $\frac{e_{j}^{\prime}}{e_{j}}$ shows the amount of growth (not progress or regress). In literature discussing evaluation of progress and regress, the following formula has been used:

$\rho=\frac{e_{p}^{\prime}-e_{p}}{e_{p}}$

In which $e_{p}^{\prime}$ and $e_{p}$, are relative efficiency of $D M U_{p}$ in period $t+1$ and $t$ respectively. We used this formula, the result was not acceptable from managerial point of view. The following example was put forward to convince us that the result is not realistic. This example considers two branches called A and B. supposes the relative efficiency of an in period $t+1$ and $t$ is 0.3 and 0.1 , so the progress is:

$\rho=\frac{0.3-p-0.1}{0.1}=2$ 
That means $200 \%$ progress.

Now consider branch $\mathrm{B}$ with relative efficiency of $0.95,0.85$ in periods $t+1$ and $t$. The progress is:

$\rho=\frac{0.95-p-0.85}{0.85}=0.1176$

From managerial point of view, performance of branch $\mathrm{B}$ is much better than performance of branch $\mathrm{A}$, since when a $D M U$ is close to frontier it is very hard to improve its performance, compared with a branch that is far from the efficiency frontier, so we were asked to modify the formula. The group had a long discussion; finally the function which is used below was suggested for this purpose. We think that this is not the only function that can be used for calculating Progress or Regress.

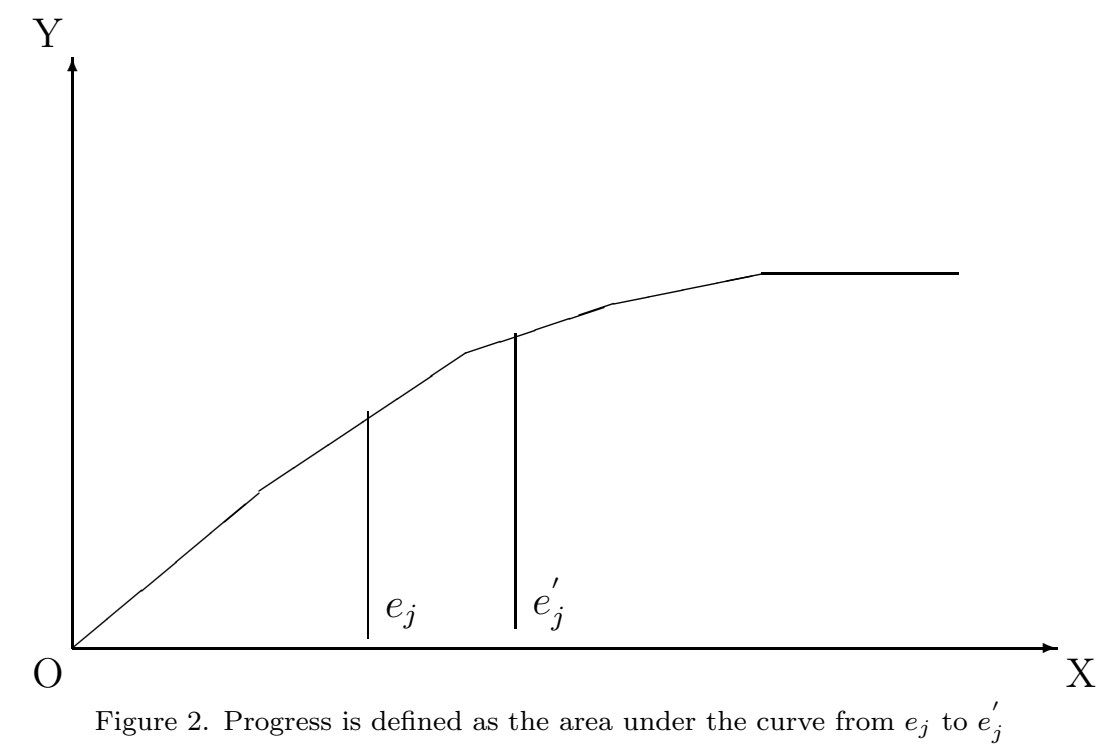

Suppose that $f$ is a function, so the amount of progress is defined as the area under the curve from $e_{j}$ to $e_{j}^{\prime}$. Therefore, we have:

The progress of $D M U_{j}=\rho=\int_{e_{j}}^{e_{j}^{\prime}} f(x) d x$

The following is one of the functions that we can propose for this purpose:

$f(x)=\frac{2 x}{x+1}$

By replacing it in (6), progress can be calculated from the following equation:

$\rho_{j}=2\left[\left(e_{j}^{\prime}-e_{j}\right)+\ln \left(\frac{e_{j}+1}{e_{j}^{\prime}+1}\right)\right]$ 
Where $e_{j}^{\prime} \leq e_{j}$, integral would be negative and it implies the regress of $D M U_{j}$, and if $e_{j}^{\prime}=e_{j}$, according to (5) we would have $\rho_{j}=0$ and there is not any progress and regress. And in case $e_{j}^{\prime} \geq e_{j}$, integral would be positive and it implies the progress of $D M U_{j}$. So we have the following Table:

Table 1. The criteria for determining progress or regress

\begin{tabular}{|c|c|}
\hline$\rho_{j}$ & Situation \\
\hline$\rho_{j}>0$ & It shows progress \\
\hline$\rho_{j}=0$ & Neither progress nor regress \\
\hline$\rho_{j}<0$ & It shows regress \\
\hline
\end{tabular}

\section{The data and analyzing the results}

This paper will assess all the branches of Bank Mellat, all over the country. Bank Mellat has 1946 branches classified into 6 different levels called, distinctive branches, $1^{\text {st }}$ degree, $2^{\text {nd }}$ degree, $3^{\text {rd }}$ degree, $4^{\text {th }}$ degree, $5^{\text {th }}$ degree, and $6^{\text {th }}$ degree, respectively.

As you see in Table 2, we allocated specific set of branches to each level.

Table 2. Classification of branches

\begin{tabular}{|c|c|}
\hline Set of branches & Branches under assessment \\
\hline Instinctive degree All the branches & Instinctive degree All the branches \\
\hline $1^{\text {st }}, 2^{\text {nd }}, 3^{r d}, 4^{t h}, 5^{t h}$, and $6^{t h}$ degrees & $1^{\text {st }}$ degree \\
\hline $2^{\text {nd }}, 3^{r d}, 4^{t h}, 5^{t h}$, and $6^{t h}$ degrees & $2^{\text {nd }}$ degree \\
\hline $3^{r d}, 4^{t h}, 5^{t h}$, and $6^{t h}$ degrees & $3^{r d}$ degree \\
\hline $4^{t h}, 5^{t h}$, and $6^{t h}$ degrees & $4^{t h}$ degree \\
\hline $5^{t h}$, and $6^{t h}$ degrees & $5^{t h}$ degree \\
\hline $6^{t h}$ degree & $6^{t h}$ degree \\
\hline
\end{tabular}

Table 3.a and Table 3.b show you the intended indicators for calculating progress and regress, respectively. The data of all the branches, during 6 months in 2002 is gathered and this duration is divided into two 3-months periods, so we compared the performance of the branches in the spring with their performance in the summer. Actually, July is compared with April, August with May, and September with June, to calculate the progress or regress of each branch. You can see the summarized results in Table 4.

Table 3.a. The result of progress

\begin{tabular}{|l|l|l|l|l|l|l|l|r|r|}
\hline Degree & \multicolumn{6}{|l|}{ First component } & \multicolumn{2}{|c|}{ Second component } & \multicolumn{2}{l|}{ accumulation } \\
\hline & 1 & 2 & 3 & 1 & 2 & 3 & 1 & 2 & 3 \\
\hline
\end{tabular}




\begin{tabular}{|c|c|c|c|c|c|c|c|c|c|c|}
20 & Distinctive & 8 & 2 & 4 & 3 & 1 & 1 & 6 & 2 & 4 \\
\hline 79 & Grade 1 & 36 & 1 & 8 & 31 & 3 & 7 & 33 & 1 & 6 \\
\hline 232 & Grade 2 & 102 & 8 & 17 & 93 & 6 & 30 & 102 & 7 & 11 \\
\hline 487 & Grade 3 & 254 & 31 & 44 & 250 & 16 & 28 & 251 & 21 & 34 \\
\hline 788 & Grade 4 & 703 & 464 & 492 & 657 & 688 & 452 & 710 & 530 & 505 \\
\hline 323 & Grade 5 & 105 & 41 & 53 & 132 & 18 & 25 & 129 & 18 & 23 \\
\hline 17 & Grade 6 & 5 & 0 & 3 & 5 & 0 & 1 & 5 & 0 & 2 \\
\hline
\end{tabular}

Table 3.b. The result of regress

\begin{tabular}{|c|c|c|c|c|c|c|c|c|c|c|}
\hline \multicolumn{2}{|r|}{ Degree } & \multicolumn{3}{|c|}{ First component } & \multicolumn{3}{|c|}{ Second component } & \multicolumn{3}{|c|}{ accumulation } \\
\hline & & 1 & 2 & 3 & 1 & 2 & 3 & 1 & 2 & 3 \\
\hline 20 & Distinctive & 12 & 18 & 16 & 17 & 19 & 19 & 14 & 18 & 16 \\
\hline 79 & Grade 1 & 43 & 78 & 71 & 48 & 76 & 72 & 46 & 1 & 73 \\
\hline 232 & Grade 2 & 130 & 224 & 215 & 139 & 226 & 202 & 130 & 225 & 221 \\
\hline 487 & Grade 3 & 231 & 454 & 439 & 236 & 470 & 458 & 235 & 465 & 452 \\
\hline 788 & Grade 4 & 84 & 323 & 292 & 130 & 100 & 335 & 78 & 358 & 383 \\
\hline 323 & Grade 5 & 188 & 272 & 261 & 191 & 305 & 298 & 194 & 305 & 300 \\
\hline 17 & Grade 6 & 12 & 17 & 12 & 12 & 17 & 16 & 12 & 17 & 15 \\
\hline
\end{tabular}

These Tables consist of two columns, the first column is the degree of the branch along with their number, and the second column we have the number of branches with progress in Table 3.a and the number of branches with regress contains branches with regress in Table 3.b. The second column in Table 3.a and Table 3.b were each divided into three columns which are correspondent to the first component, second component, and accumulation. Each of these columns also contains three smaller columns. Column 1 shows the number of branches which made progress or regress in July compared with April, column 2 shows the number of branches which made progress or regress in August compared with May, and column 3 shows the same information about September and June. The difference between the total number of branches in a given degree, with the number of branches with progress and regress, is the number of branches which made neither progress nor regress. For example, in $6^{\text {th }}$ degree, first component, third column, 3 branches showed progress and 12 branches showed regress, and as the total number of $6^{\text {th }}$ degree branches is 17 , we have 2 branches without any progress or regress in this month. According to these Tables, most of the distinctive branches showed progress in the first component, while in other degrees both components were affected equally. It shows that distinctive branches focus more on resource absorption which is the first 
component, and try to be successful in this area. While, in other degrees, the progress factor in both components (resource absorption and giving services) acts equally.

Consider that distinctive branches have particular customers such as large companies and factories and atc, so they should be more active in absorbing resources and avoid giving unnecessary services.

As can be seen in Table 3.a, in all degrees, the number of branches which show progress in July, compared with April, is much more than other months because usually at the beginning of each fiscal year (Iranian fiscal year begins in April), commercial banks experience a deflation rooted in the economic deflation of the society and it would be modified later.

Another important point in this Table is the remarkable progress of branches in $4^{\text {th }}$ degree. In Table 4 , we have the percentage of branches with progress in accumulative efficiency in different degrees. $4^{\text {th }}$ degree with $74 \%$ progress is in highest level and the lowest level belongs to $6^{\text {th }}$ degree.

Table 4. The highest level and the lowest level of progress

\begin{tabular}{|c|c|c|c|c|c|c|c|}
\hline Degree & Distinctive & Grade 1 & Grade 2 & Grade 3 & Grade 4 & Grade 5 & Grade 6 \\
\hline Percentage & $\% 60$ & $\% 51$ & $\% 52$ & $\% 63$ & $\% 74$ & $\% 53$ & $\% 41$ \\
\hline
\end{tabular}

By studying important inputs and outputs such as resources, interests, facilities and atc, the reason of high progress percentage in $3^{\text {rd }}$ and $4^{\text {th }}$ degrees were detected. We found out that there is a narrow difference between these data in moderate degrees with a higher degree, so they are motivated to improve their output and be promoted to a higher degree. While for a branch in $2^{\text {nd }}$ degree rising to a higher degree is not so easy and there is a considerable difference between its data and those of $1^{\text {st }}$ degree and that's why their motivation to improve their performance is less than moderate degrees. $5^{\text {th }}$ and $6^{\text {th }}$ degrees are in such a bad condition, maybe because of undesirable geographical and environmental situation, that are not able to compete with other degrees.

\section{Conclusion}

In this paper, a method is proposed for measuring the progress and regress in different periods of time and it has been applied to the branches of Bank Mellat. This method tried to remove the sudden changes of data in some branches to reduce the remarkable changes in progress and regress of those branches. However, the sudden changes that each branch experience at the end of the fiscal year, produces a drop in the performance of April, that's why the number of progressed branches in July is much more than April. To reduce the effect of such events on the measuring process the performance of the 
branches is assessed in different periods of time.

The results show that the progress of moderate branches is more than the others, and that's because of the competition exists between them for rising to a higher degree. But in lower levels such as $5^{\text {th }}$ and $6^{\text {th }}$ degrees, there is more regress than progress, and we wonder that if they should be allowed to continue their activities.

\section{References}

Banker R. D., A. Charnes and W. W. Cooper (1984) "Some model for estimating technical and scale inefficiencies in DEA", Management Science, Vol. 30 (9), pp. 1078-1092.

Charnes A., W. W. Cooper and E. Rhodes (1978) "Measuring the efficiency of DMUs", European Journal of Operational Research, Vol. 2, pp. 429-444.

Cook W. D., M. Hababou and H. J. H. Tuenter (2000) "Multicomponent Efficiency Measurment and Shared Inputs in Data Envelopment Analysis: An Application to Sales and Service Performance in Bank Branches", Journal of Productivity Analysis, Vol. 14, pp. 209-224.

Cooper W. W., L. M. Seiford and K. Tone (2000a), "Data Envelopment Analysis" Kluwer Academic Publication, Boston, Dordrecht, London.

Jahanshahloo G. R., F. Hosseinzadeh Lotfi, N. Shoja, G. Tohidi and S. Razavian,(2004), "The output estimation of a DMU according to improvement of its efficiency" Applied Mathematics and Computation, Vol. 147, pp. 409-413. Wei Q. L., J. Zhang and X. Zhang (2000), "An inverse DEA model for input/output estimate", European Journal of Operational Research, Vol. 121, pp. 151-163.

Received: September 25, 2005 